\title{
Fishing for Spectral Lines in High-Redshift Galaxies Lensed by Frontier Fields Clusters
}

\author{
Adi Zitrin \\ Hubble Fellow; Cahill Center for Astronomy and Astrophysics, California Institute of \\ Technology, MC 249-17, Pasadena, CA 91125, USA \\ email: adizitrin@gmail.com
}

\begin{abstract}
The Hubble Frontier Fields, together with other cluster lensing surveys with Hubble, have revealed hundreds of high-redshift $(z \gtrsim 6)$ dropout galaxy candidates. We summarize recent efforts taken to spectroscopically follow up magnified high-redshift galaxies behind those clusters and in the field, including our detection of Ly $\alpha$ emission from a redshift $z=8.68$ object.
\end{abstract}

Keywords. cosmology: observations — galaxies: high-redshift — galaxies: evolution — galaxies: formation - gravitational lensing: strong

Background It is by now well established that the Universe has become completely reionized by redshift $\sim 6$ (e.g. Fan et al. 2006). One of the main evidence for the evolving state of the intergalactic medium (IGM) at high redshift is the rapid decline observed in the visibility of Ly $\alpha 1216 \AA$ emission from photometrically-selected galaxies at $z \gtrsim 6$ due to resonant scattering by neutral Hydrogen.

Spectroscopy of high-redshift galaxies is crucial for understanding the physical properties of the first galaxies and the reionization process. Because of the scattering of Ly $\alpha$ emission, however, it is increasingly difficult to detect higher-redshift galaxies substantially above $z \sim 6$. Despite much observational effort with the largest telescopes, only about a dozen galaxies are spectroscopically confirmed above $z \sim 7$ (e.g. Ono et al. 2012, Vanzella et al. 2011, Schenker et al. 2014, Finkelstein et al. 2013, Oesch et al. 2015), and no Ly $\alpha$ had been seen above redshift $z \simeq 7.7$.

Due to the declining visibility of $\operatorname{Ly} \alpha$ at higher redshifts, the most viable route for spectroscopically studying high- $z$ galaxies is targeting alternative ultraviolet (UV) spectral lines, such as CIII] $(\lambda \lambda 1907,1909 \AA)$ and CIV $(\lambda \lambda 1548,1550 \AA)$. While typically $\sim 10$ times fainter intrinsically than Ly $\alpha$ (Stark et al. 2014), due to the increasing fractions of neutral Hydrogen those UV lines should become apparently more prominent at higher redshifts when Ly $\alpha$ is completely attenuated by the intervening neutral gas. Recently Stark et al. 2015b and Stark et al. 2015a detected CIII] in a $z \simeq 6$ and $z \simeq 7.2$ galaxies, and CIV in a $z \simeq 7.05$ galaxy, with previous Ly $\alpha$ emission known, manifesting the potential of this observational route.

Our Keck / MOSFIRE Campaign Following this success we embarked (in 2014B) on a short campaign to search for CIII] and CIV emission in galaxies lensed by Hubble Frontier Fields, and CLASH (PI: Postman) clusters. The relative high density of targets concentrated in one field thanks to the lens magnification is especially beneficial in conjunction with the multi-slit capabilities of the Multi-Object Spectrometer For InfraRed Exploration (MOSFIRE, McLean et al. 2012) on the Keck 1 telescope, to enhance chances for detection. This is particularly important, as unlike the 2-3 previous detections here we aimed to imitate future surveys without prior knowledge of the exact redshift (e.g. from $\operatorname{Ly} \alpha$ ) so that the chances for detection decrease both subjectively (it is more difficult to ascertain a few-sigma line detection and separate it from random noise) and 
statistically, given the distribution of skylines. Specifically, we targeted $\sim 20-30$ galaxies in the redshift range $\sim 6-9$, with higher priority given to brighter objects and those with better photometric redshifts. The typical integration time per field was around 2-3 hours, and up to 5 hours - reaching a $5 \sigma$ flux limit of about $1.5 \times 10^{-18} \mathrm{ergs} \mathrm{cm}^{-2} \mathrm{sec}^{-1}$. This flux limit is better than that obtained by Stark et al. in their previous detections, but here most of the objects are fainter and so in terms of equivalent width (EW) we were typically only sensitive to lines that are intrinsically stronger (higher EWs).

While no definitive line was detected in this campaign, several important conclusions were drawn: First, CIII] and CIV, albeit a promising route, are not necessarily less challenging to detect - in absence of exact redshift from other lines - than Ly $\alpha$, at least not in the probed redshift $z \sim 7$. Second, it seems that unlike previous claims that fainter objects might actually show higher EWs, one should still prefer brighter objects also for UV line detection. Last, we managed to statistically fold our non-detections into an upper limit on the typical CIII] rest-frame EW in our sample, of $\sim 26 \AA$ (typical $M_{U V} \sim-19$ ). This was the first time CIII] was - to our knowledge - searched systematically in a statistical sample of high-redshift galaxies. Our results were summarized in Zitrin et al. 2015a.

Ly $\alpha$ at $z=\mathbf{8 . 7}$ Are there other indicators that can guide us towards line detection aside for brightness? Recently, Roberts-Borsani et al. 2015 (see also Labbé et al. 2013, Smit et al. 2015), have used significantly red IRAC [3.6]-[4.5] colors indicative of strong emission lines to extract four $z>7$ galaxies from the CANDLES fields, out of which three were targeted spectroscopically. Remarkably, all three showed Ly $\alpha$ emission despite being at $z>7.5$ where the neutral Hydrogen fraction is believed to already be high enough to attenuate any such radiation. One of these three objects was targeted by us with MOSFIRE, EGS8p7, in which we detected Ly $\alpha$ at redshift $z=8.68$ (Zitrin et al. 2015b) - becoming the highest spectroscopically confirmed object to date, and potentially challenging our understanding of reionization. It remains to investigate how frequent are such objects and what are the implications on our view on reionization.

Acknowledgements AZ is indebted to the collabroators on both works covered here: Richard Ellis, Sirio Belli, Dan Stark, Rychard Bouwens, Ivo Labbé, Guido RobertsBorsani, Renske Smit, and Pascal Oesch. AZ is supported by NASA/STScI's Hubble Fellowship \#HST-HF2-51334.001-A. We are grateful for Keck Telescope observations.

\section{References}

Fan, X., Strauss, M. A., Becker, R. H., et al. 2006, AJ, 132, 117

Finkelstein, S. L., Papovich, C., Dickinson, M., et al. 2013, Nature, 502, 524

Labbé, I., Oesch, P. A., Bouwens, R. J., et al. 2013, ApJ (Letters), 777, L19

McLean, I. S., Steidel, C. C., Epps, H. W., et al. 2012, in Society of Photo-Optical Instrumenstation Engineers (SPIE) Conference Series, Vol. 8446

Oesch, P. A., van Dokkum, P. G., Illingworth, G. D., et al. 2015, ApJ (Letters), 804, L30

Ono, Y., Ouchi, M., Mobasher, B., et al. 2012, ApJ, 744, 83

Roberts-Borsani, G. W., Bouwens, R. J., Oesch, P. A., et al. 2015, arXiv, 1506.00854

Schenker, M. A., Ellis, R. S., Konidaris, N. P., \& Stark, D. P. 2014, ApJ, 795, 20

Smit, R., Bouwens, R. J., Franx, M., et al. 2015, ApJ, 801, 122

Stark, D. P., Richard, J., Siana, B., et al. 2014, MNRAS, 445, 3200

Stark, D. P., Walth, G., Charlot, S., et al. 2015a, arXiv, 1504.06881

Stark, D. P., Richard, J., Charlot, S., et al. 2015b, MNRAS, 450, 1846

Vanzella, E., Pentericci, L., Fontana, A., et al. 2011, ApJ (Letters), 730, L35

Zitrin, A., Ellis, R. S., Belli, S., \& Stark, D. P. 2015a, ApJ (Letters), 805, L7

Zitrin, A., Labbé, I., Belli, S., et al. 2015b, ApJ (Letters), 810, L12 